\title{
Combining environmental suitability and population abundances to evaluate the invasive potential of the tunicate Ciona intestinalis along the temperate South American coast
}

Stella M Januario, Sergio A Estay, Fabio A Labra, Mauricio Lima

The tunicate Ciona intestinalis is an opportunistic invader with high potential for causing economic losses in aquaculture centers. Recent phylogenetic and population genetic analysis support the existence of a genetic complex described as $C$. intestinalis with two main dominant species (sp A and B) occurring worldwide. In Chile, the species has been observed around $30^{\circ} \mathrm{S}$ of latitude, but no official reports exist for the presence of $C$. intestinalis in southern regions (above $40^{\circ} \mathrm{S}$ ), where most of the mollusk aquaculture centers are located. Here, we used occurrences from multiple invaded regions and extensive field sampling to model and validate the environmental conditions that allow the species to persist and to find the geographic areas with the most suitable environmental conditions for the spread of $C$. intestinalis in the Chilean coast. By studying the potential expansion of $C$. intestinalis southward in the Chilean Coast, we aimed to provide valuable information that might help the development of control plans before the species becomes a significant problem, especially above $40^{\circ} \mathrm{S}$. Our results highlight that, by using portions of the habitat that are apparently distinguishable, the species seem to be not only genetically distinct, but ecologically distinct as well. The two regional models fitted for sp A and for sp B showed disagreement on which sections of Chilean coastline are considered more suitable for these species. While the model for $\mathrm{sp} A$ identifies moderately to highly suitable areas between $30^{\circ}$ and $40^{\circ} \mathrm{S}$, the model for sp B classifies the areas around $45^{\circ} \mathrm{S}$ as the most appropriate. Data from field sampling show a positive linear relationship between density of $C$. intestinalis and the index of suitability for sp $A$ in aquaculture centers. Understanding the relation of the distinct species with the surrounding environment provided valuable insights about probable routes of dispersion in Chile, especially into those areas considered suitable for aquaculture activities but where the species has not yet been recorded. We discuss the implications of our findings as a useful tool to anticipate the invasion of such harmful invasive species with regard to the most relevant environmental variables. 
Combining environmental suitability and population abundances to evaluate the invasive potential of the tunicate Ciona intestinalis complex along the temperate South American coast

4 Abstract: The tunicate Ciona intestinalis is an opportunistic invader with high potential for 5 causing economic losses in aquaculture centers. In Chile, the species has been observed around

$630^{\circ} \mathrm{S}$ of latitude, but no official reports exist for the presence of $C$. intestinalis in southern regions

7 (above $40^{\circ} \mathrm{S}$ ), where most of the mollusk aquaculture centers are located. Here, we used

8 occurrences from multiple invaded regions sourced from public databases, and extensive field

9 sampling to model and validate the environmental conditions that allow the species to persist and

10 to find the geographic areas with the most suitable environmental conditions for the spread of $C$.

11 intestinalis along the Chilean coast. Our results highlight that, by using portions of the habitat

12 that are apparently distinguishable, the species seem to be not only genetically distinct, but

13 ecologically distinct as well. The two regional models fitted for sp A and for sp B showed

14 disagreement on which sections of Chilean coastline are considered more suitable for these

15 species. While the model for sp A identifies moderately to highly suitable areas between $30^{\circ}$ and

$1640^{\circ} \mathrm{S}$, the model for sp B classifies the areas around $45^{\circ} \mathrm{S}$ as the most appropriate. Data from

17 field sampling show a positive linear relationship between density of $C$. intestinalis and the index

18 of suitability for sp A in aquaculture centers. Understanding the relationship of the distinct

19 species with the surrounding environment provided valuable insights about probable routes of

20 dispersion in Chile, especially into those areas considered suitable for aquaculture activities but

21 where the species has not yet been recorded.

22 Stella M. Januario, Departamento de Ecología, Facultad de Ciencias Biológicas, Pontificia

23 Universidad Católica de Chile, Santiago, Chile. and Departamento de Ciencias Biológicas y

24 Quimicas, Facultad de Ciencias, Universidad San Sebastián, Valdivia, Chile

25 Sergio A. Estay, Instituto de Ciencias Ambientales y Evolutivas, Facultad de Ciencias.

26 Universidad Austral de Chile, Valdivia, Chile. and Center of Applied Ecology \& Sustainability

27 (CAPES), Facultad de Ciencias Biológicas, Pontificia Universidad Católica de Chile, Santiago,

28 Chile.

29 Fabio A. Labra, Centro de Investigación e Innovación para el Cambio Climático, Facultad de

30 Ciencias, Universidad Santo Tomas, Santiago, Chile.

31 Mauricio Lima, Departamento de Ecología, Pontificia Universidad Católica de Chile, Santiago,

32 Chilea and Center of Applied Ecology \& Sustainability (CAPES), Facultad de Ciencias 
33 Biológicas, Pontificia Universidad Católica de Chile, Santiago, Chile.

\section{Corresponding author}

35 Stella M. Januario*, Departamento de Ecología, Pontificia Universidad Católica de Chile,

36 Santiago, Chile.Phone 56962162006, stella.mjs@gmail.com

37 *Current Address: Departamento de Ciencias Biológicas y Químicas, Facultad de Ciencias,

38 Universidad San Sebastián, General Lagos 1163, Valdivia, Chile 


\section{Introduction}

40 Marine invertebrates are amongst the species with the highest potential for invasion and 41 damage (Capinha et al., 2012; Lee et al., 2008; Robinson et al., 2011). Most of the incursions of 42 invasive species in coastal areas are nowadays inevitable due to the worldwide traffic of marine 43 vessels (Ramsay et al., 2008; Ashelby et al., 2013), and the co-transference of organisms during 44 importation of commercially exploited species for stocking or aquaculture purposes (Locke \& 45 Hanson, 2009). Many of these opportunist species take advantage of human activity to extend 46 their distributions, and are associated with aquaculture centers, causing large damage to both 47 cultures and natural environments. This is the case of the tunicate Ciona intestinalis (Karayucel, 48 1997, Hecht \& Heasman, 1999, Uribe \& Etchepare, 2002), a sessile filter feeder that lives in 49 dense aggregations in enclosed or semi-protected marine embayments (Carver et al., 2006). Many 50 of its life history traits make this species a successful invader, including its rapid growth rates

51 (20mm/month), early maturation (8-10 weeks) and high reproductive output ( $>10000$

52 eggs/individual). In addition, it exhibits wide environmental tolerance, especially temperature 53 (Carver et al., 2003). Across its native range (North Atlantic) it is considered a dominant competitor in benthic communities, while in its exotic range it occurs as an opportunistic fouling organism on artificial substrates in harbors or in association with aquaculture equipment (Carver et al., 2006).

57 Recently, it has been discovered that the taxon $C$. intestinalis actually corresponds to a genetic 58 complex of 2 to 4 species (Suzuki et al., 2005; Iannelli et al., 2007; Zhan et al., 2010). Two of them, the species A and B are the most common forms, with the widest geographic distribution (Zhan et al., 2010). Both sp A and sp B are distributed worldwide: sp A has invaded the Pacific

61 Ocean, the Mediterranean Sea, Australia and South Africa, while sp B occupies Northern Europe, 62 including the British coastline, as well as the east coast of North America and Canada. The two 
63 remaining species, $\mathrm{C}$ and $\mathrm{D}$, are rare, and remain restricted to small areas in the Mediterranean

64 and Black Sea, respectively (Zhan et al., 2010). Recently, has been proposed that sp. A actually

65 correspond to C. robusta and sp. B to the original C. intestinalis (Brunetti et al., 2015; Pennati et

66 al. 2015). Although there have been efforts to use phenotypic traits such as body color,

67 pigmentation at the distal end of the siphons and the presence or absence of tubercles on the sides

68 of the siphons to facilitate the identification of these different species in the field (Sato et al.,

69 2012; Brunetti et al., 2015; Pennati et al. 2015), it is likely that available information regarding

70 the distribution of the species is a mix of records of the whole genetic complex. For this reason,

71 in this study we will maintain the sp. A and sp. B classification, but keeping in mind the new

72 proposal taxonomy.

73 Over the last few decades population outbreaks have been observed at many sites around the

74 world including South Africa (Hecht \& Heasman, 1999), Scotland (Karayucel, 1997), and Chile

75 (Uribe \& Etchepare, 2002). As a result, the species has become a serious biofouling problem to

76 the marine aquaculture industry (Edwards \& Leung, 2009). In particular, few years ago the

77 invasion by $C$. intestinalis in Canada was considered to be at "crisis level", and the species has

78 been considered a major marine invasive issue for the Department of Fisheries and Oceans of

79 Canada (Edwards \& Leung, 2009). Under these circumstances, understanding the ecological

80 niche of this particular species complex would provide valuable information about how they

81 manage to survive and establish dense populations in such distinct areas as the Mediterranean Sea

82 and the much colder North Atlantic Ocean.

83 Along the temperate South American ecoregion, the species has been recorded from around $30^{\circ} \mathrm{S}$

84 Lat. in the Chilean coast, in the regions of Coquimbo and Antofagasta (Castilla et al., 2005),

85 where it has been reported as being responsible for economic losses caused by damage to

86 suspended cultures of Argopecten purpuratus (Uribe \& Etchepare, 2002). According to Castilla \&

87 Neill (2009) the introduction and spread of the species into this region has been facilitated by the

88 continuous transfer of propagules and materials between aquaculture centers. However, its wide

89 physiological tolerance, reflected in its extensive world distribution (Madariaga et al., 2014),

90 might facilitate the expansion of its range along the Chilean coast. Currently there are no official

91 reports for the presence of $C$. intestinalis in southern regions (above $40^{\circ} \mathrm{S}$ ), where most of the

92 centers for the culture of molluscs are located (Norambuena \& Gonzalez, 2005), and where small

93 and medium size aquaculture centers play a key role in the economy and social interaction of 
94 local communities (Norambuena \& Gonzalez, 2005).

95 A practical way to understand and ultimately predict range expansions of invaders is by 96 characterizing the environmental conditions that are currently suitable for the persistence of a 97 given species (Pearson, 2007), and then identifying those areas where such conditions are 98 distributed in the geographic space (Colwell \& Rangel, 2009, Franklin, 2010). A group of 99 quantitative modeling approaches, known collectively as Ecological Niche Modeling (ENM) 100 have been widely used for this purpose in recent years (Soberón \& Peterson, 2005; Peterson, 101 2006; Soberón \& Nakamura, 2009; Elith \& Leathwick, 2009; Zimmermann et al., 2010; Peterson $102 \&$ Soberón, 2012). The central assumption of ENM is that the response functions estimated in 103 these models provide an effective representation of the spatial response of the species to different 104 environments (Cassini, 2011). In this sense, ENMs provide a quantitative and formal procedure to 105 106 for potential or ongoing invasions (Locke \& Hanson, 2009).

107 Despite their merits, the use of these methods in the management of invasive species requires two 108 important difficulties to be overcome. First, results from ENMs rely heavily on the assumption 109 that species are in equilibrium with the environment (Pearman et al., 2008; Colwell \& Rangel, 110 2009; Peterson, 2011). To fulfill this main assumption it is important to take into account habitat 111 similarity between ranges, aiming to ensure that the ENM analysis remains restricted to those 112 areas that present similar environmental conditions (Randin et al., 2006). In this vein, analog 113 environments in an invaded range represent those habitats inside the range of values considered

114 to quantify the native range and so, correspond to environmental conditions that have been 115 experienced by the species before the invasion; otherwise, habitat are considered non-analog, and 116 conclusions about these environments must be taken with caution (Owens et al., 2013; Fitzpatrick 117 \& Hargrove, 2009). Second, results of ecological niche models (ENMs) are usually expressed as 118 quantitative suitability indexes or as probability of presence, which are not necessarily linked to 119 population abundances, a key parameter for pest managers or conservation biologists

120 (VanDerWal et al., 2009). To overcome this second caveat, adequate modeling procedures and 121 field validation of the fitted ENMs are necessary.

122 In this study we combine ENM with extensive field sampling to provide valuable information 123 that might help the development of plans of control before these species become a significant 
124 problem. In particular we try to answer the following questions: Will the complex $C$. intestinalis

125 continue to spread in the Chilean coast, or does it already occupy most of its potential range? If it

126 continues to spread, will the spread extend to regions containing high concentration of

127 aquaculture centers?. Finally, are niche models indexes reflecting population abundances at a

128 confidence level useful for pest managers and conservation biologists? The answers to these

129 questions will provide key information for an adequate planning of prevention and control task in

130 aquaculture centers, especially in southern Chile, where these centers represent a major economic

131 activity for local communities.

\section{Methods}

133 Species occurrence data

134 Confirmed records on the occurrence of $C$. intestinalis were obtained from the Global

135 Biodiversity Information Facility (GBIF - www.gbif.org). After removing duplicate records and a

136 few records that presented obvious errors of georeference, the final dataset consisted of 776 from

137 Northern Europe (considered here as the native range) as well as 107 presences registered in

138 Canada, 98 records from the Pacific coast of the United States, 212 from Southern Europe, and

13924 records from Japan. Due to their morphological similarity, and the consequent difficulty

140 involved in the identification of each species in the field (but see Sato et al., 2012), we cannot

141 unambiguously attribute records from the public databases to the different species within the

142 genetc complex. Therefore, we decided to follow Zhan et al., (2010) and allocate the set of

143 occurrences to the species that dominates a specific area.Therefore, data from Canada and

144 Northern Europe were considered as the current distribution of $\mathrm{sp} \mathrm{B}$. The other areas represent

145 the distribution of sp A. We used occurrences from each area to calibrate single-models (hereafter

146 called "Canada model" or "Southern Europe model", etc.) and regional models (considering

147 occurrences from more than one region where each species dominates). These single and regional

148 models were then used to predict the potential distribution of $C$. intestinalis throughout the

149 Chilean coast. Original distributions were defined using a $20 \mathrm{~km}$ buffer around the reported 150 presence points.

151 Occurrences and density of C. intestinalis in the Chilean coast

152 To validate the results of our ENMs, we obtained confirmed presence of $C$. intestinalis by 
153 surveys in aquacultures centers throughout the Chilean coast. We visited the three main regions

154 where aquacultures centers are located in northern, central and southern Chile according to the

155 information provided by regional agencies of the Sernapesca (National Fishery Service). Centers

156 producing oysters, mussels, abalones and scallops were visited. Fifteen localities were sampled

157 from 27 to $43^{\circ} \mathrm{S}$. latitude. along the Chilean coast (spanning approximately 1,700 Kms) during

158 the summer seasons of the years 2013-2015. In each locality, all aquaculture centers and

159 infrastructure (docks and pilings) were visually inspected for presence of C. intestinalis.

160 Photographic records were taken and later were used to calculate the relative density of $C$.

161 intestinalis in each site. Considering that some aquaculture centers are located closer than the

162 resolution of oceanographic layers used in this study (see next section), we used average density

163 by locality in our analysis to avoid pseudo-replication. Density was expressed as the number of

164 individuals per $225 \mathrm{~cm}^{2}(15 \times 15 \mathrm{~cm}$ grid $)$.

\section{Environmental variables}

166 We chose oceanographic layers representing various quantitative environmental predictors with a 167 recognized physiological and ecological relevance for C. intestinalis (Carver et al., 2006;

168 Madariaga et al., 2014). These were Sea Surface Temperature - SST (minimum, mean, maximum 169 and range), Photosynthetically Available Radiation - PAR (mean, maximum), Salinity (mean), $\mathrm{pH}$ 170 (mean), Dissolved Oxygen - Dissox (mean), Chlorophyll A - Chlo (maximum, mean, minimum).

171 All variables were obtained from BioOracle database (Tyberghein et al., 2012) with a spatial

172 resolution of 5 arcmin (c. $9 \mathrm{~km}$ ). Most of the grids contained monthly records for the period

173 between 2002 to 2009, except PARmax and PARmean, which encompassed records from 1997 to

174 2009. The environmental layers were processed with Quantum GIS 2.6.0 to fit the extent of each 175 zone.

\section{Statistical methods}

177 The dataset was separated into separate geographic areas to build single area models (East

178 Canada, West USA, Japan, Southern Europe, Northern Europe), and regional models that

179 grouped more than one area where each species dominates. For sp A we calibrated a model with 180 records from Japan, United States and Southern Europe, and for sp B a model with occurrences 181 from Northern Europe and Canada. We did not consider models for sp C and sp D, given their 
182 lower frequencies in empirical records and more restricted geographic distributions. We then used

183 Niche Analyst (Qiao et al., 2013), to perform a Principal Component Analysis (PCA) on the

184 environmental variables and visualize the environmental space into transformed principal

185 component dimensions. The program uses a covariance-based approach to PCA calculation. We

186 used minimum volume ellipsoids around the points of occurrence to delimit, in the environmental

187 space, the conditions considered favorable for the persistence of the species. We later identified

188 the geographic areas in the Chilean Coast where those environmental conditions can be found.

189 Finally, we interpreted our results (suitability index) for those regions where the analog

190 environments ( similar conditions between the area where model was calibrated and the area of

191 projection) were similar. This is relevant especially from a management perspective, because it

192 makes easier to recognize areas with novel environments where niche model algorithms tend to

193 extrapolate predictions. We also identified those areas most suitable for aquaculture in the

194 Chilean coast (courtesy of Subsecretaria de Pesca - Subpesca). This allowed us to visualize the

195 areas under higher risk of invasion and damage, and hence with more potential for economic

196 losses.

197 Ecological niche models were fitted using Maximum Entropy Species Distribution Modelling

198 software v. 2.3 (Maxent). This is a useful method for making predictions especially when

199 incomplete information about species distribution is available. By evaluating the climate data at

200 each location where the species of interest is present, Maxent calculates a probability function

201 that describes the chances of observing a presence giving the observed distribution of the species

202 and the environmental conditions across the study area (Phillips et al., 2004; 2006). The output of

203 Maxent is a continuous variable which indicates environmental suitability. For each individual

204 model, we used a 20-fold cross-validation scheme, except for the "Japan model", where we used

205 a 17-fold cross-validation scheme. The area under the curve (AUC) statistic for the Receiver

206 Operating Characteristic (ROC) was used to measure how well each model discriminates

207 presences more accurately than a random prediction (Phillips et al., 2006). Fitted models were

208 later projected over the Chilean coast, using the same environmental variables, to identify where

209 suitable environments for $C$. intestinalis are likely to occur. The importance of each

210 environmental variable for every model was assessed by a jackknife procedure, fitting a model

211 using each variable separately and ranking them according to the test gain.

212 Finally, we evaluate the relationship between suitability values with the observed density. To do 
213 so, we follow VanDerWal et al., (2009) and use linear regression and quantile regression (90\%

214 percentile) to determine if suitability indexes successfully predict the upper limit of local

215 abundances. Goodness of fit was assessed using simple $\mathrm{R}^{2}$ values for linear regression, and

216 pseudo $\mathrm{R}^{2}$ values (Koenker \& Machado, 1999) for quantile regression.

\section{Results}

Principal component analysis of the pooled climatic data revealed three significant axes of climatic variation. The first three principal components accounted for $82.02 \%$ of the total variation in the data. The first principal component (PC1) was mainly thermal (related to SSTmean and SSTmin), whereas PC2 was related to SSTrange and the three Chlorophyll measures. Finally, PC3 was mainly related to salinity and $\mathrm{pH}$. Interestingly, the PCA split the populations into two groups (Fig 1). The first cluster includes mainly populations from Japan, Southern Europe and West USA. The other group embraces both, Northern Europe and populations from Canada. The climatic separation into both groups reflects the distribution of the two most common species that compose the genetic complex of $C$. intestinalis. Once the environmental space was defined using the first three principal components, we estimated the environmental niche by fitting a minimum volume ellipsoid around the presence points of each species. All points inside the ellipsoid represent environments with favorable conditions for the species in the original region. We projected the minimum volume ellipsoid in Chilean territory to identify analog environments (habitats inside the range of values found in the original region). Analog environments for sp.A are almost four times more common in Chile than those for sp.B according to the projections from the minimum volume ellipsoid (Fig 2).

Models for original distributions were significantly better than random and performed well according to AUC (table I). The lowest AUC was obtained for the model of Northern Europe $(A U C=0.82)$, while the highest was obtained for the model of West USA $(A U C=0.94)$. For the regional models, the observed AUC values were 0.87 and 0.80 for sp A and sp B, respectively. Only regional models are shown. All the remaining models, obtained with single modelling areas as well as the projections with non-analog environments may be found in Figures S1-2.

240 The two regional models (sp A and sp B) showed strong disagreement for portions of Chile that 
242 showed that central and southern regions (30 $-40^{\circ} \mathrm{S}$, Fig 2) seem more suitable for establishment

243 than the peripheral regions of Chile (below $25^{\circ}$ and above $43^{\circ} \mathrm{S}$ ). Only a few patches of suitable

244 habitats are found in the most extreme southern region of the continent, around the $53^{\circ} \mathrm{S}$. The

245 model for the sp B revealed that the most suitable habitats are located around the $45^{\circ} \mathrm{S}$ (Fig 2).

246 Additional areas near $53^{\circ} \mathrm{S}$ were also classified with high suitability index, the same as observed

247 with the model of sp A.

248 Based on Jacknife analyses for regional models the distribution of sp A was most influenced by 249 mean Salinity and Chlorophyll A (both mean and minimum). For sp B, Sea Surface Temperature

250 (minimum) and Photosynthetically Available Radiation (PAR) (mean) were important

251 contributors (Table 1). The most important variables also varied in the single models, as in

252 Canada where mean values of Chlorophyll A correspond to the variable that contributed most to

253 the model, while in Southern Europe, Salinity and pH were the most important variables (Table 254 I).

255 Our field sampling detected $C$. intestinalis in most aquaculture centers examined in 15 localities 256 between $27^{\circ}$ and $43^{\circ} \mathrm{S}$ latitude (Fig 2). To the best of our knowledge, this is the first report of $C$.

257 intestinalis infestations south of $30^{\circ} \mathrm{S}$ latitude on the temperate South American pacific coast, 258 specifically in mussel, oyster and abalone farms. Density of C.intestinalis showed a clear north259 south pattern, with higher densities in northern Chile and low densities in the South.

260 The relative density of the species in aquaculture centers revealed contrasting results. For sp A. 261 the plot showed a positive, linear relationship between observed density and the suitability index $262\left(\mathrm{R}^{2}=0.26\right.$, Fig 3$)$, and a strong relationship at the $90 \%$ percentile (pseudo $\mathrm{R}^{2}=0.55$, Fig 3 ). For sp $263 \mathrm{~B}$, the relationship was completely absent, and for the upper limit was negative, which makes no 264 sense in this context $\left(\mathrm{R}^{2}=0.0\right.$, pseudo $\mathrm{R}^{2}=0.08$, Fig 3$)$.

\section{Discussion}

Our results showed that, when considering the distribution of each species of the genetic complex of $C$. intestinalis (sp A and B), the species seem to be not only genetically distinct, but also appear to be ecologically distinct. Our analyses separated the species into two main groups based on their environmental preferences. Interestingly, the PCA analysis of environmental 
270

271

272

273

274

275

276

277

278

279

280

281

282

283

284

285

286

287

288

289

290

291

292

293

294

295

296

297

298

299

conditions for the presence points was coherent with the genetic separation among the species within the complex. In consequence, not considering these differences in environmental models might lead to imprecise conclusions about the potential distribution of the species outside their original range.

The first principal component is mostly influenced by mean and minimum values of sea surface temperature. These variables grouped occurrences from Northern Europe and Canada where the minimum sea temperature reach values close to $5^{\circ} \mathrm{C}$ and separated them from observations obtained in areas like Japan, east USA and the Mediterranean Sea, where minimum values of temperature are much higher $\left(11.7^{\circ} \mathrm{C}\right.$, based on layers provided by BioOracle). Although the taxon is recognized by its wide tolerance to temperature variation (Carver et al., 2006)), our results suggest a distinct range of preferences at least for the two most common species. By isolating the two groups, we could improve the power of our predictions by restricting the projections of the models to the geographic areas where the environmental conditions are analog to those where the models were calibrated. We took this precaution because some model algorithms tend to extrapolate projections beyond the range of environmental values used to calibrate the models and end up identifying high values of suitability even in conditions where most of the species are unlikely to survive (Owens et al., 2013).

The two regional models (for sp A and for sp B) showed disagreement for portions of Chile that are considered suitable for the maintenance of the species. Projections of the model of sp A showed that climate in Chile is moderately to highly suitable, especially around the $30^{\circ}$ and $40^{\circ} \mathrm{S}$, while for sp B, the areas around the $45^{\circ} \mathrm{S}$ are the most appropriate. Also, the model for sp B predicted several suitable areas beyond the extent of the current invaded range of $C$. intestinalis in the Chilean coast.

We interpreted projections in the Chilean coast only to those analog environments to those where the models were calibrated. For the model of sp A, it resulted in a void in the projection layer between the $34^{\circ}-37^{\circ} \mathrm{S}$ and between $46^{\circ}-51^{\circ} \mathrm{S}$. These areas are known as strong upwelling centers, and they also receive important influxes of freshwater (Atkinson et al., 2002; Dávila et al., 2002). Both factors may generate particular conditions that are not shared by the other areas where the species has been found. In any case, our survey confirmed the presence of $C$. intestinalis in some aquaculture centers around the $36^{\circ} \mathrm{S}$ Lat. Initial colonization in this zone 
300

301

302

303

304

305

306

307

308

309

310

311

312

313

314

315

316

317

318

319

320

321

322

323

324

325

326

327

328

329

330

331

started, most likely, with specimens that benefited from the exchange of equipment between aquaculture centers from other parts of the country where the species has already established dense populations (IV Región, around $30^{\circ} \mathrm{S}$ ). For sp B, analog environments, identified by the minimum volume ellipsoid, are just found southern $40^{\circ} \mathrm{S}$, which means that only above this latitude coastal environmental conditions are similar to those the species found in Canada and Northern Europe.

The model for the sp A seems to capture quite well the current distribution of $C$. intestinalis in Chile, especially in the area of Coquimbo $\left(29^{\circ} \mathrm{S} 71^{\circ} \mathrm{W}\right)$ where the biofouling effect of the species has been a major problem on the suspended cultures of scallops(Uribe \& Etchepare, 2002). Indeed, it is assumed the species first arrived in the area brought by Japanese boats which transported the personal and equipment used in the implementation of the first centers for the culture of scallop (Madariaga et al., 2014). The exchange of boats and equipment facilitated the spread of the species northward, where it can be found in dense populations also associated with cultures of scallops, especially during the summer. Later, the species has the potential to spread to the area close to Puerto Montt (around $41^{\circ} \mathrm{S}$ ), although in a much lower density. Although the origin of the specimens found in the Chilean coast have been tentatively attributed to Japan, which could correspond to the sp A, the single model adjusted with occurrences from Japan identified no analog environments in the Chilean coast (Online Resource 1,2). This model is probably biased by the few points of occurrence that we obtained from the public registry for the area of Japan, and also to the proximity of the points. It means, our single model for Japan is probability characterizing only a narrow portion of the possible niche for the species. The model for sp B considered suitable areas situated outside the present distribution in Chile, and where most of the aquaculture centers are located. In some areas, the species is already established but still at low densities. Indeed, most of the mariculturalists that we could contact in southern regions (above $40^{\circ} \mathrm{S}$ ) did not recognize $C$. intestinalis as a real threat to their cultures, which contrasted with the response from mariculturalists from the northern-central Chile, who could readily identify $C$. intestinalis and view it as a real threat. Anecdotally, farm workers only recognize $C$. intestinalis correctly in the northern region of Chile. In the farms located at southern Chile, workers misidentify $C$. intestinalis with early stages of Pyura chilensis. Hence, southern regions must be considered a priority in future plans of management and control, which should include programs to provide adequate training to local mariculturalists to improve the changes of early detection of new invasions and to prevent contamination due sharing equipment between 
farms.

333 Madariaga et al., (2014) used information from unifactorial experiments to assess the tolerance to

334 light, salinity and temperature of individuals collected in the Region of Coquimbo ( $\left.30^{\circ} \mathrm{S}\right)$. They

335 also compiled data from literature to associate performance (mixing several metrics as mortality,

336 filtration rate and particle retention efficiency) with salinity and temperature. These authors

337 suggest that the species is physiologically capable of tolerating the wide range of physical

338 conditions found in Chile, suggesting the whole Chilean coastline may be considered at high risk.

339 However, data compiled in this study was not separated into the different species within the

340 complex (i.e. sp A, B , C or D); therefore, the observed performance cannot be assigned to any

341 species in particular or be considered representative of the species already present in Chile. To the

342 best of our knowledge, there are no available studies that compare the physiological tolerance of

343 each of these four species independently. Nevertheless, our results suggest the two dominant

344 species of the genetic complex are probably using different portions of the environmental space.

345 The Jacknife analyses that included each variable alone, reinforced the separation of the two

346 species in the environmental space. For sp A, Salinity and Chlorophyll A (mean; minimum) are

347 the most important variables. For sp B, Sea Surface Temperature (minimum) and

348 Photosynthetically Available Radiation (PAR) were important contributors. Previous studies have

349 reported that temperature is an important cue for sexual maturation, spawning and recruitment in

350 C. intestinalis (Dybern, 1967; Marin et al., 1987; Carver et al., 2003; Howes et al., 2007). For

351 instance, in Scandinavian and subarctic populations, where temperature rarely exceeds $8^{\circ} \mathrm{C}$, the

352 generational time is 2-3 years and individuals are reproductively mature at the first year (Dybern,

353 1965; Dybern, 1967). For Japan and the warmer Mediterranean where temperatures are always

354 above $10^{\circ} \mathrm{C}$, the generational times vary between 3 and 6 months and sexual maturity is reached

355 after 1 to 2 months, depending on the season (Yamaguchi, 1975). The optimal salinity for

356 Mediterranean populations (35\%), is much higher than would normally experienced by northern

357 Atlantic coastal populations (Marin et al., 1987). Lambert and Lambert (1998) reported that $C$.

358 intestinalis populations on floating docks in southern California harbors were vulnerable to

359 pulses of low salinity. On the other hand, Dybern (1967) found that the lower salinity limit for

360 adults and developmental stages in Scandinavian populations was 11\%o. Such differences might

361 emphasize the capability of the species to survive under a broad range of conditions, but can also

362 reinforce the implications from our results, that each species of the genetic complex might be 
363 using different portions of the niche. This information is crucial, especially when using ENMs to 364 study how species colonize new environments (Sax et al., 2007) and whether they retain their 365 climatic niche in a new range (Pearman et al., 2008).

366 Here, we observed a linear relationship between relative density and suitability index for sp A, 367 especially in relation to the upper limit of local abundances (Fig 3, 90\% quantile regressions). 368 VanDerWal et al., (2009) pointed out that suitability indexes reflect potential abundance, but 369 other factors may prevent the species attain this potential. In our case, the index explains $56 \%$ of 370 the variation on the upper limit of the population density among aquacultures centers, which 371 means that those centers located in areas classified as possessing a high suitability index could 372 sustain more abundant populations. For $\mathrm{sp} \mathrm{B}$, the same relationship was null. In our case, we can 373 speculate the reason why some localities do not show the expected higher abundance could be 374 associated to environmental variables outside the model or acting at a small scale such as

375 turbidity or management activities in aquaculture centers; however, finding the exact reason will 376 require new studies.

377 According to the National Fishery Service, the areas around the $42^{\circ} \mathrm{S}$ encompass most of the 378 centers for the culture of molluscs in Chile. Such areas must be of high priority for control plans. 379 Some areas above the $50^{\circ} \mathrm{S}$ may also serve as potential habitat for $C$. intestinalis from a strictly 380 climatic perspective. However, they are not considered suitable for aquaculture, so it is not clear 381 whether they could support wild population of the species. In fact, Dumont et al. (2009) suggest 382 that despite the well-established populations on artificial structures, the species appears unable to 383 colonize natural communities due to predation pressure from native benthic species, especially 384 the rock shrimp Rhyncocinetes typus. On the other hand, competition with the native ascidian 385 Pyura chilensis seems unimportant in natural benthic communities exposed to natural predation 386 (Dumont et al. 2009).

387 In the case of the few places where marine invaders have been successfully controlled, first 388 actions typically occurred in the early stages of invasion, right after establishment and initial 389 spread (Edwards \& Leung, 2009). The higher a population size, the longer the species will 390 persist, and eradication will be no longer a management option (Lockwood et al., 2013). In this 391 context, our results might help the Chilean regulatory agencies to identify which areas must be 392 prioritized in eventual control plans. Considering the potential risk to southern Chile, 
393

394

395

396

397

398

399

400

401

402

403

404

405

406

407

408

409

410

411

412 Peterson. recorded. management of $C$. intestinalis invasions should concentrate on the reduction of the probability of introduction due to contaminated individual vessels (Drake \& Lodge, 2004), controlling the number of potential invaders on transport or recreational boats. Controlling the exchange of contaminated equipment between aquaculture centers might also reduce the fortuitous spread of the species.

Risk maps are in worldwide demand for management purposes, however they are clearly dependent on the type of occurrence data used (Therriault \& Herborg, 2008). If it is possible to link suitability and abundance, ENMs may turn into a very powerful tool in the management of invasive species. Even if management measures have not been able to eliminate biological invasions, slowing the rate of invasion or spread of an established species has considerable value (Ruiz \& Carlton, 2003). In our case, ENMs have allowed us to understand the relation of the distinct species that conform the genetic complex known as $C$. intestinalis with their surrounding environment providing valuable insights about probable routes of dispersion in Chile, especially into those areas considered adequate for aquaculture activities and where the species has not been

\section{Acknowledgments}

We thank Daniela Lopez, Melissa Pavez, Kennia Morales, Roger Sepúlveda for their valuable help during field work, and to all anonymous mariculturalists that kindly received us and shared their experience with the field team. This paper was much improved by the comments of Dr. AT Peterson. 


\section{References}

415

416

417

418

419

420

421

422

423

424

425

426

427

428

429

430

431

432

433

434

435

436

437
Ashelby CW, Johnson ML, De Grave S. 2013. The global invader Palaemon macrodactylus (Decapoda, Palaemonidae): an interrogation of records and a synthesis of data. Crustaceana 86:594-624.

Atkinson LP, Valle-Levinson A, Figueroa D, De Pol-Holz R, Gallardo VA, Schneider W, Blanco JL, Schmidt M. 2002. Oceanographic observations in Chilean coastal waters between Valdivia and Concepción. J Geophys Res 107:1-14.

Brunetti R, Gissi C, Pennati R, Caicci R, Gasparini F, Manni L. 2015 Morphological evidence that the molecularly determined Ciona intestinalis type A and type B are different species: Ciona robusta and Ciona intestinalis. J Zoolog Syst Evol Res 53: 186-193

Capinha C, Anastácio P, Tenedório JA. 2012. Predicting the impact of climate change on the invasive decapods of the Iberian inland waters: an assessment of reliability. Biol Invasions 14:1737-1751.

Carver C, Chisholm A, Mallet AL. 2003. Strategies to mitigate the impact of Ciona intestinalis (L.) biofouling on shellfish production. J Shellfish Res 22:621-632.

Carver CE, Mallet AL, Vercaemer B. 2006. Biological synopsis of the solitary tunicate Ciona intestinalis. 55.

Cassini MH. 2011. Ecological principles of species distribution models: the habitat matching rule. J Biogeogr 38:2057-2065.

Castilla JC, Neill PE. 2009. Marine bioinvasions in the Southeastern Pacific : status, ecology, economic impacts, conservation and management. In: Rilov and Crooks (eds). Biological invasions in marine ecosystems. Springer. p. 439-457.

Castilla JC, Uribe M, Bahamonde N, Clarke M, Desqueyroux-Faúndez R, Kong I, Moyano H, Rozbaczylo N, Santelices B, Valdovinos C, Zavala P. 2005. Down under the southeastern 
Pacific: marine non-indigenous species in Chile. Biol Invasions 7:213-232.

439

440

441

442

443

444

445

446

447

448

449

450

451

452

453

454

455

456

457

458

459

460

461

462

463

464

465

466

467

468

469

470

471

472

473

474

475

476

477

478
Colwell RK, Rangel TF. 2009. Hutchinson's duality: the once and future niche. Proc Natl Acad Sci U S A 106 Suppl :19651-8.

Dávila PM, Figueroa D, Müller E. 2002. Freshwater input into the coastal ocean and its relation

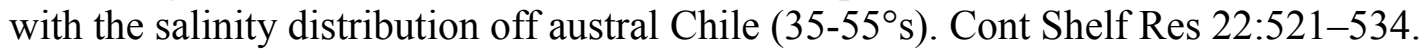

Drake JM, Lodge DM. 2004. Global hot spots of biological invasions: evaluating options for ballast-water management. Proc Biol Sci 271:575-80.

Dumont CP, Urriago JD, Abarca A, Gaymer CF, Thiel M. 2009. The native rock shrimp Rhynchocinetes typus as a biological control of fouling in suspended scallop cultures. Aquaculture 292:74-79.

Dybern BI. 1965. The life cycle of Ciona intestinalis (L.) f. typica in relation to the environmental temperature. Oikos 16:109-131.

Dybern BI. 1967. The distribution and salinity tolerance of Ciona intestinalis (L.) F. typica with special reference to the waters around Southern Scandinavia. Ophelia 4:207-226.

Edwards PK, Leung B. 2009. Re-evaluating eradication of nuisance species: invasion of the tunicate Ciona intestinalis. Front Ecol Environ 7:326-332.

Elith J, Leathwick JR. 2009. Species Distribution Models: ecological explanation and prediction across space and time. Annu Rev Ecol Evol Syst 40:677-697.

Fitzpatrick MC, Hargrove WW. 2009. The projection of species distribution models and the problem of non-analog climate. Biodivers Conserv 18:2255-2261.

Franklin J. 2010. Mapping Species Distributions: Spatial Inference and Prediction. Cambridge University Press

Hecht T, Heasman K. 1999. The culture of Mytilus galloprovincialis in South Africa and the carrying capacity of mussel farming in Saldanha Bay. World Aquac Rouge 30:50-55.

Howes S, Herbinger CM, Darnell P, Vercaemer B. 2007. Spatial and temporal patterns of recruitment of the tunicate Ciona intestinalis on a mussel farm in Nova Scotia, Canada. J Exp Mar Bio Ecol 342:85-92.

Iannelli F, Pesole G, Sordino P, Gissi C. 2007. Mitogenomics reveals two cryptic species in Ciona intestinalis. Trends Genet 23:419-22.

Koenker, R., \& Machado, JA. 1999. Goodness of fit and related inference processes for quantile regression. JASA 94:1296-1310.

Lambert CC, Lambert G. 1998. Non-indigenous ascidians in southern California harbors and marinas. Mar Biol 130:675-688.

Lee H, Reusser DA, Olden JD, Smith SS, Graham J, Burkett V, Dukes JS, Piorkowski RJ, McPhedran J. 2008. Integrated monitoring and information systems for managing aquatic invasive species in a changing climate. Conserv Biol 22:575-84.

Locke A, Hanson JM. 2009. Rapid response to non-indigenous species. 1. Goals and history of rapid response in the marine environment. Aquat Invasions 4:237-247.

Madariaga DJ, Rivadeneira MM, Tala F, Thiel M. 2014. Environmental tolerance of the two invasive species Ciona intestinalis and Codium fragile: their invasion potential along a temperate coast. Biol Invasions 16:2507-2527. 
Marin MG, Bressan M, Beghi L, Brunetti R. 1987. Thermo-haline tolerance of Ciona intestinalis (L. 1767) at different developmental stages. Cah Biol Mar 28:47-57.

Norambuena R, Gonzalez L. 2005. National aquaculture sector overview Fact Sheets - Chile. FAO, Rome.

Owens HL, Campbell LP, Dornak LL, Saupe EE, Barve N, Soberón J, Ingeloff K, Lira-Noriega A, Hensz CM, Myers CE, Peterson AT. 2013. Constraints on interpretation of ecological niche models by limited environmental ranges on calibration areas. Ecol Modell 263:10-18.

Pearman PB, Guisan A, Broennimann O, Randin CF. 2008. Niche dynamics in space and time. Trends Ecol Evol 23:149-58.

Pearson RG. 2007. Species Distribution Modeling for conservation educators and practitioners. Lessons in Conservation 3:54-89

Pennati R, Ficetola GF, Brunetti R, Caicci F, Gasparini F, Griggio F, Sato A, Stach T, KaulStrehlow S, Gissi C, Manni L. 2015 Morphological Differences between Larvae of the Ciona intestinalis Species Complex: Hints for a Valid Taxonomic Definition of Distinct Species. PlosONE e0122879.

Peterson AT, Soberón J. 2012. Species distribution modeling and ecological niche modeling: Getting the Concepts Right. Nat a Conserv 10:102-107.

Peterson AT. 2006. Uses and requirements of ecological niche models and related distributional models. Biodivers Informatics 3:59-72.

Peterson AT. 2011. Ecological niche conservatism: a time-structured review of evidence. J Biogeogr 38:817-827.

Phillips SJ, Anderson RP, Schapire RE. 2006. Maximum entropy modeling of species geographic distributions. Ecol Modell 190:231-259.

Phillips SJ, Dudík M, Schapire RE. 2004. A maximum entropy approach to species distribution modeling. Twenty-first Int. Conf. Mach. Learn. - ICML '04. ACM Press, New York, New York, USA, p 83

Qiao H, Escobar LE, Soberón J, Campbell L, Peterson AT. 2014. NicheA. Version 1.2 beta. Available at: http://biodiversity- informatics-training.org/software-data-sources/nichea/ (accessed on December 2014).

Ramsay A, Davidson J, Landry T, Arsenault G. 2008. Process of invasiveness among exotic tunicates in Prince Edward Island, Canada. Biol Invasions 10:1311-1316.

Randin CF, Dirnböck T, Dullinger S, Zimmermann NE, Zappa M, Guisan A. 2006. Are nichebased species distribution models transferable in space? J Biogeogr 33:1689-1703.

Robinson LM, Elith J, Hobday AJ, Pearson RG, Kendall BE, Possingham HP, Richarson AJ. 2011. Pushing the limits in marine species distribution modelling: lessons from the land present challenges and opportunities. Glob Ecol Biogeogr 20:789-802.

Ruiz GM, Carlton J. 2003. Invasive Species: Vectors And Management Strategies. Island Press.

Sato A, Satoh N, Bishop JDD. 2012. Field identification of "types" A and B of the ascidian Ciona intestinalis in a region of sympatry. Mar Biol 159:1611-1619.

Sax DF, Stachowicz JJ, Brown JH, Bruno JF, Dawson MN, Gaines SD, Grosberg RK, Hastings A, Holt RD, Mayfield MM et al. 2007. Ecological and evolutionary insights from species 
invasions. Trends Ecol Evol 22:465-71.

Soberón J, Nakamura M. 2009. Niches and distributional areas : Concepts, methods , and assumptions. PNAS 106:19644-19650.

Soberón J, Peterson AT. 2005. Interpretation of models of fundamental ecological niches and species' distributional areas. Biodivers Informatics 2:1-10.

Therriault TW, Herborg L. 2008. A qualitative biological risk assessment for vase tunicate Ciona intestinalis in Canadian waters : using expert knowledge. J Mar Sci 65:781-787.

Tyberghein L, Verbruggen H, Pauly K, Troupin C, Mineur F, De Clerck O. 2012. Bio-ORACLE: a global environmental dataset for marine species distribution modelling. Glob Ecol Biogeogr 21:272-281.

530

Uribe E, Etchepare I. 2002. Effects of biofouling by Ciona intestinalis on suspended culture of Argopecten purpuratus. Bull. AAC. 3:93-95.

VanDerWal J, Shoo LP, Johnson CN, Williams SE. 2009. Abundance and the environmental niche: environmental suitability estimated from niche models predicts the upper limit of local abundance. Am Nat 174:282-291.

535

Yamaguchi M. 1975. Growth and reproductive cycles of the marine fouling ascidians Ciona intestinalis, Styela plicata, Botrylloides violaceus and Leptoclinum mitsukurii at AburatsuboMoroiso Inlet (central Japan). Mar Biol 29:253-259.

Zhan A, Macisaac HJ, Cristescu ME. 2010. Invasion genetics of the Ciona intestinalis species complex: from regional endemism to global homogeneity. Mol Ecol 19:4678-94.

Zimmermann NE, Edwards TC, Graham CH, Pearman PB, Svenning J. 2010. New trends in species distribution modelling. Ecography (Cop) 33:985-989.

\section{Figure captions}

543 Figure 1. Principal component analysis for the environmental variables at the presence points.

544 PC1 and PC2 scores are shown. Colors represent different populations of $C$. intestinalis. Scores at

545 PC1 markedly separate distributions of sp A (Japan, Southern Europe and USA) and sp B

546 (Northern Europe and Canada).

547 Figure 2. Sampling points and projections of the potential distribution of $C$. intestinalis sp A and $548 \mathrm{sp}$ B on Chilean coast. In the first map, red dots show the localities sampled into the three major 549 aquacultural regions of Chile. Non analog environments are projections on Chilean environments 550 that may be or may not be represented at locations used to fit the model. Analog environments are 551 projections only on those Chilean environments that are represented at locations used to fit the 552 model.

553 Figure 3. Regressions between suitability indices from ENMs and observed densities in the field

554 for sp A and sp B. Dark lines represent linear regressions, gray lines represent 90\% quantile 
555 regressions. 


\section{Table 1 (on next page)}

Model's results 
1 Table I. Values of the average test AUC for the replicate runs for each single and regional

2 model. Environmental variables with the highest gain when used in isolation are shown.

3

\begin{tabular}{lll}
\hline \multicolumn{1}{c}{ Model } & AUC & \multicolumn{1}{c}{ Variable with the highest gain } \\
\hline Canada & 0.834 & Chlorophyll A (mean) \\
Japan & 0.889 & Chlorophyll A (mean; maximum) \\
West USA & 0.939 & Photosynthetically Available Radiation (PAR)(maximum) \\
Southern Europe & 0.892 & Salinity; Ph \\
Northern Europe & 0.817 & Sea Surface Temperature (minimum); PAR (mean) \\
\hline sp A & 0.873 & Salinity; Chlorophyll A (mean; minimum) \\
sp B & 0.804 & Sea Surface Temperature (minimum); PAR (mean)
\end{tabular}


Figure 1 (on next page)

PCA plot 


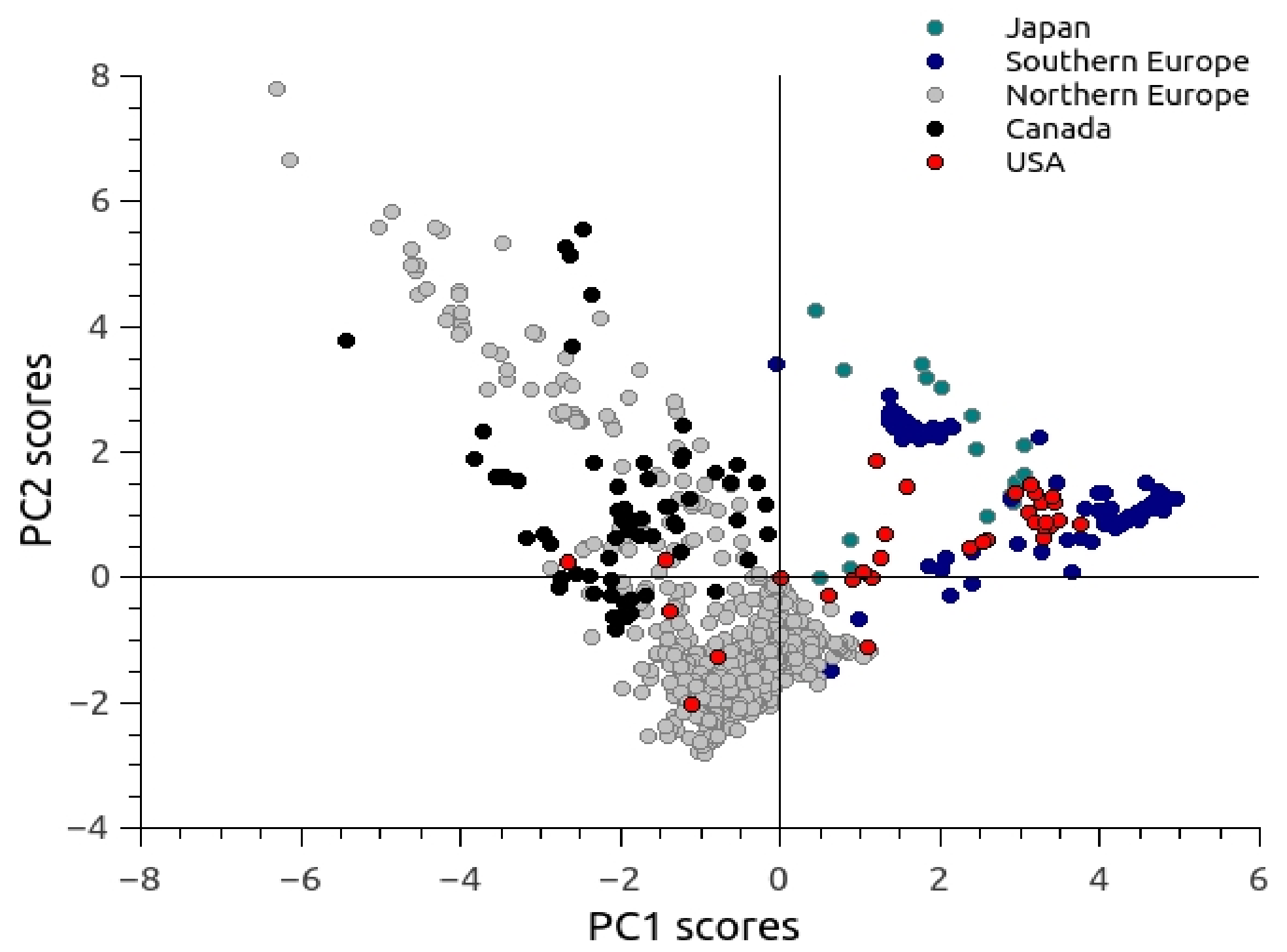




\section{Figure 2 (on next page)}

\section{Maps}




$$
\angle C l=
$$


Figure 3 (on next page)

regression plots 

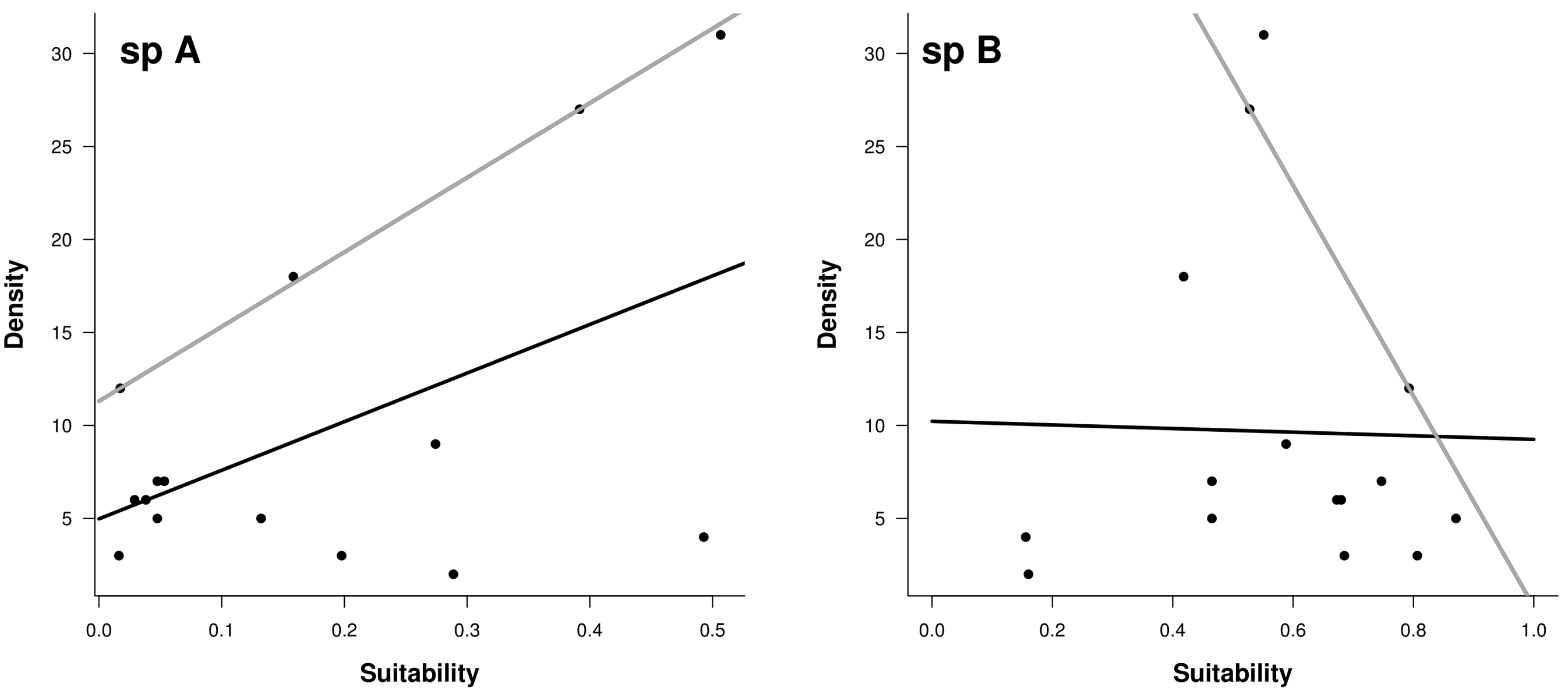